\title{
A study of coupled systems of mixed order fractional differential equations and inclusions with coupled integral fractional boundary conditions
}

Sotiris K. Ntouyas ${ }^{1,2}$ and Hamed H. Al-Sulami ${ }^{2 *}$

${ }^{*}$ Correspondence:

hhaalsalmi@kau.edu.sa

${ }^{2}$ Nonlinear Analysis and Applied

Mathematics (NAAM)—Research

Group, Department of Mathematics,

Faculty of Science, King Abdulaziz

University, Jeddah, Saudi Arabia

Full list of author information is

available at the end of the article

\section{Springer}

\begin{abstract}
In this work we investigate existence and uniqueness of solutions for new coupled systems of mixed order fractional differential equations and inclusions supplemented with coupled nonlocal fractional boundary conditions. We apply the Leray-Schauder alternative and the Banach contraction mapping principle to obtain the existence and uniqueness results, while in the multi-valued case we use the nonlinear alternative for Kakutani maps and Covitz and Nadler's fixed point theorem.
\end{abstract}

MSC: 34A08; 34B15; 34A60

Keywords: Fractional differential equations; Fractional differential inclusions; System; Mixed order; Existence; Fixed point

\section{Introduction}

Fractional differential equations recently attracted much attention in view of their extensive applications in engineering, physics, chemistry, biology, and other fields [1-3]. In particular, boundary value problems of fractional differential equations and inclusions subject to a variety of boundary conditions have been studied by many authors, for instance, see [4-11] and the references cited therein. Coupled systems of fractional-order differential equations also constitute an interesting area of investigation as such systems appear in the mathematical modeling of many phenomena like synchronization of chaotic systems [12-14], anomalous diffusion [15], ecological effects [16], disease models [17-19], etc. For some recent works on coupled systems of fractional-order differential equations, we refer the reader to the articles [20-29].

Differential inclusions are found to be of great utility in studying dynamical systems and stochastic processes. For some recent results on boundary value problems for fractional differential inclusions, see [30-33].

(c) The Author(s) 2020. This article is licensed under a Creative Commons Attribution 4.0 International License, which permits use, sharing, adaptation, distribution and reproduction in any medium or format, as long as you give appropriate credit to the original author(s) and the source, provide a link to the Creative Commons licence, and indicate if changes were made. The images or other third party material in this article are included in the article's Creative Commons licence, unless indicated otherwise in a credit line to the material. If material is not included in the article's Creative Commons licence and your intended use is not permitted by statutory regulation or exceeds the permitted use, you will need to obtain permission directly from the copyright holder. To view a copy of this licence, visit http://creativecommons.org/licenses/by/4.0/. 
Recently, in [34], the authors studied a boundary value problem of coupled Caputo type fractional differential inclusions of the form:

$$
\begin{cases}{ }^{c} D^{\alpha} u(t) \in F(t, u(t), v(t)), & t \in[0, T], 1<\alpha \leq 2, \\ { }^{c} D^{\beta} v(t) \in G(t, u(t), v(t)), & t \in[0, T], 1<\beta \leq 2, \\ u(0)=v_{1} v(T), & u^{\prime}(0)=v_{2} v^{\prime}(T), \\ v(0)=\mu_{1} u(T), & v^{\prime}(0)=\mu_{2} u^{\prime}(T),\end{cases}
$$

where ${ }^{c} D^{\alpha},{ }^{c} D^{\beta}$ denote the Caputo fractional derivatives of orders $\alpha$ and $\beta$ respectively, $F, G:[0, T] \times \mathbb{R} \times \mathbb{R} \rightarrow \mathcal{P}(\mathbb{R})$ are given multi-valued maps, $\mathcal{P}(\mathbb{R})$ is the family of all nonempty subsets of $\mathbb{R}$, and $v_{i}, \mu_{i}, i=1,2$, are real constants with $v_{i} \mu_{i} \neq 1, i=1,2$. By applying standard fixed point theorems for multi-valued maps, some new existence results for the given problem are derived when the multi-valued maps involved in the given problem have convex as well as non-convex values.

In this work, motivated by [34], we consider the following systems of Caputo and Riemann-Liouville type mixed order coupled fractional differential equations and inclusions:

$$
\left\{\begin{array}{l}
{ }^{c} D^{\alpha} x(t)=f(t, x(t), y(t)), \quad t \in[0, T], 0<\alpha \leq 1 \\
{ }^{\mathrm{RL}} D^{\beta} y(t)=g(t, x(t), y(t)), \quad t \in[0, T], 1<\beta \leq 2,
\end{array}\right.
$$

and

$$
\left\{\begin{array}{l}
{ }^{c} D^{\alpha} x(t) \in F(t, x(t), y(t)), \quad t \in[0, T], 0<\alpha \leq 1 \\
{ }^{\mathrm{RL}} D^{\beta} y(t) \in G(t, x(t), y(t)), \quad t \in[0, T], 1<\beta \leq 2,
\end{array}\right.
$$

subject to the following coupled fractional boundary conditions:

$$
\left\{\begin{array}{l}
x(0)=\lambda^{c} D^{p} y(\eta), \quad 0<p<1, \\
y(0)=0, \quad y(T)=\gamma I^{q} x(\xi)
\end{array}\right.
$$

where ${ }^{c} D^{\alpha},{ }^{c} D^{p}$ are the Caputo fractional derivatives of order $\alpha$ and $p$ respectively, ${ }^{\mathrm{RL}} D^{\beta}$ is the Riemann-Liouville fractional derivative of order $\beta, I^{q}$ is the Riemann-Liouville fractional integral of order $q, f, g:[0, T] \times \mathbb{R} \times \mathbb{R} \rightarrow \mathbb{R}, F, G:[0, T] \times \mathbb{R} \times \mathbb{R} \rightarrow \mathcal{P}(\mathbb{R})$ are given continuous functions, $\mathcal{P}(\mathbb{R})$ is the family of all nonempty subsets of $\mathbb{R}, \eta, \xi \in(0, T)$, and $\lambda, \gamma \in \mathbb{R}$.

Here we emphasize that the proposed single and multi-valued problems include:

- fractional derivatives of different orders $\alpha \in(0,1]$ and $\beta \in(1,2]$;

- the first and second equations in the given systems are respectively of Caputo and Riemann-Liouville types;

- the boundary conditions are of nonlocal type and contain both fractional derivatives and integrals.

The objective of the present work is to establish existence criteria for solutions of problems (1.1)-(1.3) and (1.2)-(1.3). For single-valued system (1.1)-(1.3), we rely on the LeraySchauder alternative and the Banach contraction mapping principle to obtain the exis- 
tence and uniqueness results, which are presented in Sect. 3. Section 4 contains the existence results for convex and non-convex valued multi-valued maps $F$ and $G$ involved in multi-valued system (1.2)-(1.3), which are respectively derived with the aid of the nonlinear alternative for Kakutani maps and Covitz and Nadler's fixed point theorem. The background material related to our work is outlined in Sect. 2. Here we remark that the tools of the fixed point theory employed in our analysis are standard, however their exposition to the problems at hand is new.

\section{Preliminaries}

Let us begin this section with some basic definitions of multi-valued maps [35, 36].

Let $(\mathcal{X},\|\cdot\|)$ be a normed space and that $\mathcal{P}_{c l}(\mathcal{X})=\{\mathcal{Y} \in \mathcal{P}(\mathcal{X}): \mathcal{Y}$ is closed $\}, \mathcal{P}_{c p, c}(\mathcal{X})=$ $\{\mathcal{Y} \in \mathcal{P}(\mathcal{X}): \mathcal{Y}$ is compact and convex $\}$.

A multi-valued map $\mathcal{G}: \mathcal{X} \rightarrow \mathcal{P}(\mathcal{X})$ is

(a) convex (closed) valued if $\mathcal{G}(x)$ is convex (closed) for all $x \in \mathcal{X}$;

(b) upper semi-continuous (u.s.c.) on $\mathcal{X}$ if, for each $x_{0} \in \mathcal{X}$, the set $\mathcal{G}\left(x_{0}\right)$ is a nonempty closed subset of $\mathcal{X}$ and if, for each open set $N$ of $\mathcal{X}$ containing $\mathcal{G}\left(x_{0}\right)$, there exists an open neighborhood $\mathcal{N}_{0}$ of $x_{0}$ such that $G\left(\mathcal{N}_{0}\right) \subseteq N$;

(c) lower semi-continuous (l.s.c.) if the set $\{y \in X: \mathcal{G}(y) \cap B \neq \emptyset\}$ is open for any open set $B$ in $E$;

(d) completely continuous if $\mathcal{G}(\mathbb{B})$ is relatively compact for every $\mathbb{B} \in \mathcal{P}_{b}(\mathcal{X})=\{\mathcal{Y} \in \mathcal{P}(\mathcal{X}): \mathcal{Y}$ is bounded $\}$

A multi-valued map $\mathcal{G}:[a, b] \rightarrow \mathcal{P}_{c l}(\mathbb{R})$ is said to be measurable if, for every $y \in \mathbb{R}$, the function $t \longmapsto d(y, \mathcal{G}(t))=\inf \{|y-z|: z \in \mathcal{G}(t)\}$ is measurable.

A multi-valued map $\mathcal{G}:[a, b] \times \mathbb{R}^{2} \rightarrow \mathcal{P}(\mathbb{R})$ is said to be Carathéodory if (i) $t \longmapsto G(t, x, y)$ is measurable for each $x, y \in \mathbb{R}$ and (ii) $(x, y) \longmapsto \mathcal{G}(t, x, y)$ is upper semicontinuous for almost all $t \in[a, b]$.

Further a Carathéodory function $\mathcal{G}$ is called $L^{1}$-Carathéodory if (i) for each $\rho>0$, there exists $\varphi_{\rho} \in L^{1}\left([a, b], \mathbb{R}^{+}\right)$such that $\|\mathcal{G}(t, x, y)\|=\sup \{|v|: v \in \mathcal{G}(t, x, y)\} \leq \varphi_{\rho}(t)$ for all $x, y \in \mathbb{R}$ with $\|x\|,\|y\| \leq \rho$ and for a.e. $t \in[a, b]$.

Next, we outline some preliminary concepts of fractional calculus.

Definition 2.1 The fractional integral of order $\sigma$ with the lower limit zero for a function $\zeta$ is defined as

$$
I^{\sigma} \zeta(t)=\frac{1}{\Gamma(\sigma)} \int_{0}^{t} \frac{\zeta(s)}{(t-s)^{1-\sigma}} d s, \quad t>0, \sigma>0,
$$

provided the right-hand side is point-wise defined on $[0, \infty)$, where $\Gamma(\cdot)$ is the gamma function, which is defined by $\Gamma(\sigma)=\int_{0}^{\infty} t^{\sigma-1} e^{-t} d t$.

Definition 2.2 The Riemann-Liouville fractional derivative of order $\sigma>0, n-1<\sigma<n$, $n \in \mathbb{N}$, is defined as follows:

$$
D_{0+}^{\sigma} \zeta(t)=\frac{1}{\Gamma(n-\sigma)}\left(\frac{d}{d t}\right)^{n} \int_{0}^{t}(t-s)^{n-\sigma-1} \zeta(s) d s
$$

where the function $\zeta$ has absolutely continuous derivative up to order $(n-1)$. 
Definition 2.3 The Caputo derivative of order $\sigma$ for a function $\zeta:[0, \infty) \rightarrow \mathbb{R}$ can be written as

$$
{ }^{c} D_{0+}^{\sigma} \zeta(t)=D_{0+}^{\sigma}\left(\zeta(t)-\sum_{k=0}^{n-1} \frac{t^{k}}{k !} \zeta^{(k)}(0)\right), \quad t>0, n-1<\sigma<n
$$

In the rest of the paper, we use ${ }^{c} D^{\sigma}$ instead of ${ }^{c} D_{0_{+}}^{\sigma}$ for the sake of convenience.

Remark 2.4 If $\zeta \in C^{n}[0, \infty)$, then

$$
{ }^{c} D^{\sigma} \zeta(t)=\frac{1}{\Gamma(n-\sigma)} \int_{0}^{t} \frac{\zeta^{(n)}(s)}{(t-s)^{\sigma+1-n}} d s=I^{n-\sigma} \zeta^{(n)}(t), \quad t>0, n-1<\sigma<n .
$$

The following auxiliary lemma, which concerns the linear variant of system (1.1), plays a key role in the sequel.

Lemma 2.5 Let $\phi, h \in C([0, T], \mathbb{R})$. Then the solution of the linear fractional differential system

$$
\left\{\begin{array}{l}
{ }^{c} D^{\alpha} x(t)=\phi(t), \quad t \in[0, T], 1<\alpha \leq 2 \\
{ }^{\mathrm{RL}} D^{\beta} y(t)=h(t), \quad t \in[0, T], 1<\beta \leq 2, \\
x(0)=\lambda D^{p} y(\eta), \\
y(0)=0, \quad y(T)=\gamma I^{q} x(\xi),
\end{array}\right.
$$

is equivalent to the system of integral equations

$$
x(t)=I^{\alpha} \phi(t)+\frac{\lambda}{\Lambda}\left[-T^{\beta-1} I^{\beta-p} h(\eta)+\frac{\Gamma(\beta)}{\Gamma(\beta-p)} \eta^{\beta-p-1}\left(\gamma I^{q+\alpha} \phi(\xi)-I^{\beta} h(T)\right)\right]
$$

and

$$
y(t)=I^{\beta} h(t)+\frac{t^{\beta-1}}{\Lambda}\left[I^{\beta} h(T)-\gamma I^{q+\alpha} \phi(\xi)-\lambda \gamma \frac{\xi^{q}}{\Gamma(1+q)} I^{\beta-p} h(\eta)\right],
$$

where it is assumed that

$$
\Lambda:=T^{\beta-1}+\lambda \gamma \frac{\Gamma(\beta) \xi^{q} \eta^{\beta-p-1}}{\Gamma(1+q) \Gamma(\beta-p)} \neq 0 .
$$

Proof Applying the Riemann-Liouville operators $I^{\alpha}$ and $I^{\beta}$ to the Caputo and RiemannLiouville fractional differential equations respectively in (2.1) and using the composition laws of fractional order integral and differential operators [2], we obtain

$$
x(t)=I^{\alpha} \phi(t)+c_{0} \quad \text { and } \quad y(t)=I^{\beta} h(t)+c_{1} t^{\beta-1}+c_{2} t^{\beta-2},
$$

where $c_{0}, c_{1}, c_{2}$ are arbitrary constants.

By the boundary conditions of (2.1) in (2.5), we get $c_{2}=0$ and a system of algebraic equations in the unknown constants $c_{0}$ and $c_{1}$ :

$$
c_{0}-\lambda \frac{\Gamma(\beta)}{\Gamma(\beta-p)} \eta^{\beta-p-1} c_{1}=\lambda I^{\beta-p} h(\eta)
$$




$$
\gamma \frac{\xi^{q}}{\Gamma(1+q)} c_{0}-T^{\beta-1} c_{1}=I^{\beta} h(T)-\gamma I^{q+\alpha} \phi(\xi) .
$$

Solving the above system, we get

$$
c_{0}=\frac{\lambda}{\Lambda}\left[-T^{\beta-1} I^{\beta-p} h(\eta)+\frac{\Gamma(\beta)}{\Gamma(\beta-p)} \eta^{\beta-p-1}\left(\gamma I^{q+\alpha} \phi(\xi)-I^{\beta} h(T)\right)\right]
$$

and

$$
c_{1}=\frac{1}{\Lambda}\left[I^{\beta} h(T)-\gamma I^{q+\alpha} \phi(\xi)-\lambda \gamma \frac{\xi^{q}}{\Gamma(1+q)} I^{\beta-p} h(\eta)\right] .
$$

Substituting the values of $c_{0}, c_{1}, c_{2}$ in (2.5), we get solutions (2.2) and (2.3). We can prove the converse of the lemma by direct computation. The proof is completed.

\section{Main results}

\subsection{Single-valued system (1.1)-(1.3)}

Let $X=\{x(t) \mid x(t) \in C([0, T], \mathbb{R})\}$ be the Banach space endowed with the norm $\|x\|=$ $\sup \{|x(t)|, t \in[0, T]\}$. Also let the product space $(X \times X,\|(x, y)\|)$ be the Banach space equipped with norm $\|(x, y)\|=\|x\|+\|y\|$.

We define an operator $\mathcal{H}: X \times X \rightarrow X \times X$ by

$$
\mathcal{H}(x, y)(t)=\left(\begin{array}{l}
\mathcal{H}_{1}(x, y)(t) \\
\mathcal{H}_{2}(x, y)(t)
\end{array}\right)
$$

where

$$
\mathcal{H}_{1}(x, y)(t)=I^{\alpha} \widehat{f}(t)+\frac{\lambda}{\Lambda}\left[-T^{\beta-1} I^{\beta-p} \widehat{g}(\eta)+\frac{\Gamma(\beta)}{\Gamma(\beta-p)} \eta^{\beta-p-1}\left(\gamma I^{q+\alpha} \widehat{f}(\xi)-I^{\beta} \widehat{g}(T)\right)\right]
$$

and

$$
\mathcal{H}_{2}(x, y)(t)=I^{\beta} \widehat{g}(t)+\frac{t^{\beta-1}}{\Lambda}\left[I^{\beta} \widehat{g}(T)-\gamma I^{q+\alpha} \widehat{f}(\xi)-\lambda \gamma \frac{\xi^{q}}{\Gamma(1+q)} I^{\beta-p} \widehat{g}(\eta)\right]
$$

and $\widehat{f}(t)=f(t, x(t), y(t)), \widehat{g}(t)=g(t, x(t), y(t))$. For convenience, we set the notations:

$$
\begin{aligned}
& M_{1}=\frac{T^{\alpha}}{\Gamma(1+\alpha)}+\frac{1}{|\Lambda|}|\lambda||\gamma| \frac{\Gamma(\beta)}{\Gamma(\beta-p)} \frac{\eta^{\beta-p-1} \xi^{q+\alpha}}{\Gamma(q+\alpha+1)}, \\
& M_{2}=\frac{T^{\beta-1} \eta^{\beta-p-1}|\lambda|}{|\Lambda|}\left[\frac{T \Gamma(\beta)}{\Gamma(\beta-p) \Gamma(\beta+1)}+\frac{\eta}{\Gamma(1+\beta)}\right], \\
& M_{3}=\frac{T^{\beta-1}|\gamma| \xi^{q+\alpha}}{|\Lambda| \Gamma(q+\alpha+1)}, \\
& M_{4}=\frac{T^{\beta}}{\Gamma(1+\beta)}\left(1+\frac{T^{\beta-1}}{|\Lambda|}\right)+\frac{T^{\beta-1}}{|\Lambda|}|\lambda||\gamma| \frac{\xi^{q} \eta^{\beta-p}}{\Gamma(1+q) \Gamma(\beta-p+1)} .
\end{aligned}
$$

Our first existence result is based on the Leray-Schauder alternative [37, p. 4].

Theorem 3.1 Assume that: 
$\left(A_{1}\right) f, g:[0, T] \times \mathbb{R} \times \mathbb{R} \rightarrow \mathbb{R}$ are continuous functions and that there exist real constants $k_{i}, \gamma_{i} \geq 0(i=0,1,2)$ with $k_{0}>0, \gamma_{0}>0$ such that, $\forall x_{i} \in \mathbb{R}(i=1,2)$,

$$
\begin{aligned}
& \left|f\left(t, x_{1}, x_{2}\right)\right| \leq k_{0}+k_{1}\left|x_{1}\right|+k_{2}\left|x_{2}\right|, \\
& \left|g\left(t, x_{1}, x_{2}\right)\right| \leq \gamma_{0}+\gamma_{1}\left|x_{1}\right|+\gamma_{2}\left|x_{2}\right| .
\end{aligned}
$$

If

$$
\left(M_{1}+M_{3}\right) k_{1}+\left(M_{2}+M_{4}\right) \gamma_{1}<1 \text { and }\left(M_{1}+M_{3}\right) k_{2}+\left(M_{2}+M_{4}\right) \gamma_{2}<1
$$

where $M_{i}, i=1,2,3,4$, are given by (3.2)-(3.5), then system (1.1)-(1.3) has at least one solution on $[0, T]$.

Proof Firstly we show that the operator $\mathcal{H}: X \times X \rightarrow X \times X$ defined by (3.1) is completely continuous. Notice that continuity of the operator $\mathcal{H}$ follows from that of the functions $f$ and $g$.

Let $\Omega \subset X \times X$ be bounded. Then there exist positive constants $L_{1}$ and $L_{2}$ such that $|f(t, x(t), y(t))| \leq L_{1},|g(t, x(t), y(t))| \leq L_{2}, \forall(x, y) \in \Omega$. Then, for any $(x, y) \in \Omega$, we have

$$
\begin{aligned}
\left|\mathcal{H}_{1}(x, y)(t)\right| \leq & \frac{T^{\alpha}}{\Gamma(1+\alpha)} L_{1}+\frac{|\lambda|}{|\Lambda|}\left[T^{\beta-1} \frac{\eta^{\beta-p}}{\Gamma(\beta-p+1)} L_{2}\right. \\
& \left.+|\lambda| \frac{\Gamma(\beta)}{\Gamma(\beta-p)} \eta^{\beta-p-1}\left(\frac{T^{\beta}}{\Gamma(1+\beta)} L_{2}+|\gamma| \frac{\xi^{q+\alpha}}{\Gamma(q+\alpha+1)} L_{1}\right)\right] \\
= & M_{1} L_{1}+M_{2} L_{2},
\end{aligned}
$$

which implies that

$$
\left\|\mathcal{H}_{1}(x, y)\right\| \leq M_{1} L_{1}+M_{2} L_{2}
$$

In a similar way, we can find that

$$
\left\|\mathcal{H}_{2}(x, y)\right\| \leq M_{3} L_{1}+M_{4} L_{2}
$$

From the above inequalities we conclude that the operator $\mathcal{H}$ is uniformly bounded, since $\|\mathcal{H}(x, y)\| \leq\left(M_{1}+M_{3}\right) L_{1}+\left(M_{2}+M_{4}\right) L_{2}$.

Next, we show that $\mathcal{H}$ is equicontinuous. Let $t_{1}, t_{2} \in[0, T]$ with $t_{1}<t_{2}$. Then we have

$$
\begin{aligned}
& \left|\mathcal{H}_{1}\left(x\left(t_{2}\right), y\left(t_{2}\right)\right)-\mathcal{H}_{1}\left(x\left(t_{1}\right), y\left(t_{1}\right)\right)\right| \\
& \quad \leq L_{1}\left|\frac{1}{\Gamma(\alpha)} \int_{0}^{t_{2}}\left(t_{2}-s\right)^{\alpha-1} d s-\frac{1}{\Gamma(\alpha)} \int_{0}^{t_{1}}\left(t_{1}-s\right)^{\alpha-1} d s\right| \\
& \quad \leq \frac{L_{1}}{\Gamma(\alpha)}\left\{\int_{0}^{t_{1}}\left[\left(t_{2}-s\right)^{\alpha-1}-\left(t_{1}-s\right)^{\alpha-1}\right] d s+\int_{t_{1}}^{t_{2}}\left(t_{2}-s\right)^{\alpha-1} d s\right\} \\
& \quad \leq \frac{L_{1}}{\Gamma(\alpha+1)}\left[2\left(t_{2}-t_{1}\right)^{\alpha}+\left|t_{2}^{\alpha}-t_{1}^{\alpha}\right|\right] .
\end{aligned}
$$


Analogously, we can obtain

$$
\begin{aligned}
& \left|\mathcal{H}_{2}\left(x\left(t_{2}\right), y\left(t_{2}\right)\right)-\mathcal{H}_{2}\left(x\left(t_{1}\right), y\left(t_{1}\right)\right)\right| \\
& \leq L_{2}\left[\frac{T^{\beta}}{\Gamma(1+\beta)}+|\lambda||\gamma| \frac{\xi^{q} \eta^{\beta-p}}{\Gamma(1+q) \Gamma(\beta-p+1)}\right] \frac{t_{2}^{\beta-1}-t_{1}^{\beta-1}}{|\Lambda|} \\
& \quad+L_{1} \frac{|\gamma| \xi^{q+\alpha}}{\Gamma(q+\alpha+1)} \frac{t_{2}^{\beta-1}-t_{1}^{\beta-1}}{|\Lambda|}+\frac{L_{2}}{\Gamma(\beta+1)}\left[2\left(t_{2}-t_{1}\right)^{\beta}+\left|t_{2}^{\beta}-t_{1}^{\beta}\right|\right] .
\end{aligned}
$$

Thus the operator $\mathcal{H}(x, y)$ is equicontinuous. In view of the foregoing arguments, we deduce that the operator $\mathcal{H}(x, y)$ is completely continuous.

Finally, it will be verified that the set $\mathcal{Z}=\{(x, y) \in X \times X \mid(x, y)=\theta \mathcal{H}(x, y), 0 \leq \theta \leq 1\}$ is bounded. Let $(x, y) \in \mathcal{Z}$ with $(x, y)=\theta \mathcal{H}(x, y)$. For any $t \in[0, T]$, we have

$$
x(t)=\theta \mathcal{H}_{1}(x, y)(t), \quad y(t)=\theta \mathcal{H}_{2}(x, y)(t) .
$$

Then

$$
\begin{aligned}
|x(t)| & \leq M_{1}\left(k_{0}+k_{1}|x|+k_{2}|y|\right)+M_{2}\left(\gamma_{0}+\gamma_{1}|x|+\gamma_{2}|y|\right) \\
& =M_{1} k_{0}+M_{2} \gamma_{0}+\left(M_{1} k_{1}+M_{2} \gamma_{1}\right)|x|+\left(M_{1} k_{2}+M_{2} \gamma_{2}\right)|y|,
\end{aligned}
$$

and

$$
\begin{aligned}
|y(t)| & \leq M_{3}\left(k_{0}+k_{1}|x|+k_{2}|y|\right)+M_{4}\left(\gamma_{0}+\gamma_{1}|x|+\gamma_{2}|y|\right) \\
& =M_{3} k_{0}+M_{4} \gamma_{0}+\left(M_{3} k_{1}+M_{4} \gamma_{1}\right)|x|+\left(M_{3} k_{2}+M_{4} \gamma_{2}\right)|y| .
\end{aligned}
$$

In consequence, we have

$$
\|x\| \leq M_{1} k_{0}+M_{2} \gamma_{0}+\left(M_{1} k_{1}+M_{2} \gamma_{1}\right)\|x\|+\left(M_{1} k_{2}+M_{2} \gamma_{2}\right)\|y\|
$$

and

$$
\|y\| \leq M_{3} k_{0}+M_{4} \gamma_{0}+\left(M_{3} k_{1}+M_{4} \gamma_{1}\right)\|x\|+\left(M_{3} k_{2}+M_{4} \gamma_{2}\right)\|y\|,
$$

which imply that

$$
\begin{aligned}
\|x\|+\|y\| \leq & \left(M_{1}+M_{3}\right) k_{0}+\left(M_{2}+M_{4}\right) \gamma_{0}+\left[\left(M_{1}+M_{3}\right) k_{1}+\left(M_{2}+M_{4}\right) \gamma_{1}\right]\|x\| \\
& +\left[\left(M_{1}+M_{3}\right) k_{2}+\left(M_{2}+M_{4}\right) \gamma_{2}\right]\|y\| .
\end{aligned}
$$

Thus we have

$$
\|(x, y)\| \leq \frac{\left(M_{1}+M_{3}\right) k_{0}+\left(M_{2}+M_{4}\right) \gamma_{0}}{M_{0}},
$$

where $M_{0}=\min \left\{1-\left[\left(M_{1}+M_{3}\right) k_{1}+\left(M_{2}+M_{4}\right) \gamma_{1}\right], 1-\left[\left(M_{1}+M_{3}\right) k_{2}+\left(M_{2}+M_{4}\right) \gamma_{2}\right]\right\}$, which establishes that the set $\mathcal{Z}$ is bounded. Thus, by the Leray-Schauder alternative [37], the 
operator $\mathcal{H}$ has at least one fixed point. Hence system (1.1)-(1.3) has at least one solution. The proof is complete.

The uniqueness of solutions for problem (1.1)-(1.3) is proved in the next theorem via Banach's contraction mapping principle.

Theorem 3.2 Assume that:

$\left(A_{2}\right) f, g:[0, T] \times \mathbb{R} \times \mathbb{R} \rightarrow \mathbb{R}$ are continuous functions and that there exist positive constants $\ell_{1}$ and $\ell_{2}$ such that, for all $t \in[0, T]$ and $x_{i}, y_{i} \in \mathbb{R}, i=1,2$, we have

$$
\begin{aligned}
& \left|f\left(t, x_{1}, x_{2}\right)-f\left(t, y_{1}, y_{2}\right)\right| \leq \ell_{1}\left(\left|x_{1}-y_{1}\right|+\left|x_{2}-y_{2}\right|\right), \\
& \left|g\left(t, x_{1}, x_{2}\right)-g\left(t, y_{1}, y_{2}\right)\right| \leq \ell_{2}\left(\left|x_{1}-y_{1}\right|+\left|x_{2}-y_{2}\right|\right) .
\end{aligned}
$$

Then system (1.1)-(1.3) has a unique solution on $[0, T]$, provided that

$$
\left(M_{1}+M_{3}\right) \ell_{1}+\left(M_{2}+M_{4}\right) \ell_{2}<1
$$

where $M_{i}, i=1,2,3,4$, are given by (3.2)-(3.5).

Proof Put $\sup _{t \in[0, T]} f(t, 0,0)=N_{1}<\infty, \sup _{t \in[0, T]} g(t, 0,0)=N_{2}<\infty$ and choose a positive number $r$ such that

$$
r>\frac{\left(M_{1}+M_{3}\right) N_{1}+\left(M_{2}+M_{4}\right) N_{2}}{1-\left(M_{1}+M_{3}\right) \ell_{1}-\left(M_{2}+M_{4}\right) \ell_{2}} .
$$

Then we show that $\mathcal{H} B_{r} \subset B_{r}$, where $B_{r}=\{(x, y) \in X \times X:\|(x, y)\| \leq r\}$ and $\mathcal{H}$ is defined by (3.1).

By assumption $\left(A_{2}\right)$, for $(u, v) \in B_{r}, t \in[0, T]$, we have

$$
\begin{aligned}
|f(t, x(t), y(t))| & \leq|f(t, x(t), y(t))-f(t, 0,0)|+|f(t, 0,0)| \\
& \leq \ell_{1}(|x(t)|+|y(t)|)+N_{1} \\
& \leq \ell_{1}(\|x\|+\|y\|)+N_{1} \leq \ell_{1} r+N_{1}
\end{aligned}
$$

and

$$
|g(t, x(t), y(t))| \leq \ell_{2}(\|x\|+\|y\|)+N_{2} \leq \ell_{2} r+N_{2} .
$$

In consequence, we obtain

$$
\begin{aligned}
\left|\mathcal{H}_{1}(x, y)(t)\right| & \\
\leq & \frac{T^{\alpha}}{\Gamma(1+\alpha)}\left(\ell_{1} r+N_{1}\right)+\frac{|\lambda|}{|\Lambda|}\left[T^{\beta-1} \frac{\eta^{\beta-p}}{\Gamma(\beta-p+1)}\left(\ell_{2} r+N_{2}\right)\right. \\
& \left.\quad+|\lambda| \frac{\Gamma(\beta)}{\Gamma(\beta-p)} \eta^{\beta-p-1}\left(\frac{T^{\beta}}{\Gamma(1+\beta)}\left(\ell_{2} r+N_{2}\right)+|\gamma| \frac{\xi^{q+\alpha}}{\Gamma(q+\alpha+1)}\left(\ell_{1} r+N_{1}\right)\right)\right] \\
= & \left(\ell_{1} r+N_{1}\right) M_{1}+\left(\ell_{2} r+N_{2}\right) M_{2} \\
= & \left(M_{1} \ell_{1}+M_{2} \ell_{2}\right) r+M_{1} N_{1}+M_{2} N_{2},
\end{aligned}
$$


which implies that

$$
\left\|\mathcal{H}_{1}(x, y)\right\| \leq\left(M_{1} \ell_{1}+M_{2} \ell_{2}\right) r+M_{1} N_{1}+M_{2} N_{2} .
$$

In the same way, we can find that

$$
\left\|\mathcal{H}_{2}(x, y)\right\| \leq\left(M_{3} \ell_{1}+M_{4} \ell_{2}\right) r+M_{3} N_{1}+M_{4} N_{2} .
$$

From the above inequalities, it follows that

$$
\|\mathcal{H}(x, y)\| \leq\left[\left(M_{1}+M_{3}\right) \ell_{1}+\left(M_{2}+M_{4}\right) \ell_{2}\right] r+\left(M_{1}+M_{3}\right) N_{1}+\left(M_{2}+M_{4}\right) N_{2} \leq r .
$$

Next, for $\left(x_{2}, y_{2}\right),\left(x_{1}, y_{1}\right) \in X \times X$ and for any $t \in[0, T]$, we get

$$
\begin{aligned}
& \left|\mathcal{H}_{1}\left(x_{2}, y_{2}\right)(t)-\mathcal{H}_{1}\left(x_{1}, y_{1}\right)(t)\right| \\
& \leq \frac{T^{\alpha}}{\Gamma(1+\alpha)} \ell_{1}\left(\left\|x_{2}-x_{1}\right\|+\left\|y_{2}-y_{1}\right\|\right) \\
& \quad+\frac{|\lambda|}{|\Lambda|}\left[T^{\beta-1} \frac{\eta^{\beta-p}}{\Gamma(\beta-p+1)} \ell_{2}\left(\left\|x_{2}-x_{1}\right\|+\left\|y_{2}-y_{1}\right\|\right)\right. \\
& \quad+|\lambda| \frac{\Gamma(\beta)}{\Gamma(\beta-p)} \eta^{\beta-p-1}\left(\frac{T^{\beta}}{\Gamma(1+\beta)} \ell_{2}\left(\left\|x_{2}-x_{1}\right\|+\left\|y_{2}-y_{1}\right\|\right)\right. \\
& \left.\left.\quad+|\gamma| \frac{\xi^{q+\alpha}}{\Gamma(q+\alpha+1)} \ell_{1}\left(\left\|x_{2}-x_{1}\right\|+\left\|y_{2}-y_{1}\right\|\right)\right)\right] \\
& \leq\left(M_{1} \ell_{1}+M_{2} \ell_{2}\right)\left(\left\|x_{2}-x_{1}\right\|+\left\|y_{2}-y_{1}\right\|\right),
\end{aligned}
$$

which leads to

$$
\left\|\mathcal{H}_{1}\left(x_{2}, y_{2}\right)-\mathcal{H}_{1}\left(x_{1}, y_{1}\right)\right\| \leq\left(M_{1} \ell_{1}+M_{2} \ell_{2}\right)\left(\left\|x_{2}-x_{1}\right\|+\left\|y_{2}-y_{1}\right\|\right) .
$$

Similarly, one can obtain

$$
\left\|\mathcal{H}_{2}\left(x_{2}, y_{2}\right)(t)-\mathcal{H}_{2}\left(x_{1}, y_{1}\right)\right\| \leq\left(M_{3} \ell_{1}+M_{4} \ell_{2}\right)\left(\left\|x_{2}-x_{1}\right\|+\left\|y_{2}-y_{1}\right\|\right) .
$$

From (3.7) and (3.8), we deduce that

$$
\left\|\mathcal{H}\left(x_{2}, y_{2}\right)-\mathcal{H}\left(x_{1}, y_{1}\right)\right\| \leq\left[\left(M_{1}+M_{3}\right) \ell_{1}+\left(M_{2}+M_{4}\right) \ell_{2}\right]\left(\left\|x_{2}-x_{1}\right\|+\left\|y_{2}-y_{1}\right\|\right) .
$$

Since $\left(M_{1}+M_{3}\right) \ell_{1}+\left(M_{2}+M_{4}\right) \ell_{2}<1$, therefore, $\mathcal{H}$ is a contraction. So, by Banach's contraction mapping principle, the operator $\mathcal{H}$ has a unique fixed point, which corresponds to a unique solution of problem (1.1)-(1.3). This completes the proof.

Example 3.3 Consider the following system of fractional boundary value problem:

$$
\begin{cases}{ }^{c} D^{1 / 2} x(t)=\frac{1}{4(t+2)^{2}} \frac{|x(t)|}{1+|x(t)|}+1+\frac{1}{t^{3}+32} \sin ^{2} y(t), & t \in[0,1], \\ \mathrm{RL}^{3 / 2} x(t)=\frac{1}{32 \pi} \sin (2 \pi x(t))+\frac{|y(t)|}{16(1+|y(t)|)}+\frac{1}{2}, & t \in[0,1], \\ u(0)=\sqrt{3} D^{1 / 2} y\left(\frac{1}{3}\right), & \\ y(0)=0, \quad y(1)=\sqrt{2} I^{1 / 2} x\left(\frac{1}{2}\right) . & \end{cases}
$$


Here, $\alpha=1 / 2, \lambda=\sqrt{3}, p=1 / 2, \eta=1 / 3, \beta=3 / 2, \gamma=\sqrt{2}, q=1 / 2, \xi=1 / 2$, and $f(t, x, y)=$ $\frac{1}{4(t+2)^{2}} \frac{|x|}{1+|x|}+1+\frac{1}{t^{3}+32} \sin ^{2} y$ and $g(t, x, y)=\frac{1}{32 \pi} \sin (2 \pi x)+\frac{|y|}{16(1+|y|)}+\frac{1}{2}$. Note that $\mid f\left(t, x_{1}, y_{1}\right)-$ $f\left(t, x_{2}, y_{2}\right)\left|\leq \frac{1}{16}\right| x_{1}-x_{2}\left|+\frac{1}{16}\right| y_{1}-y_{2}|| g,\left(t, x_{1}, y_{1}\right)-g\left(t, x_{2}, y_{2}\right)\left|\leq \frac{1}{16}\right| x_{1}-x_{2}\left|+\frac{1}{16}\right| y_{1}-y_{2} \mid$. Using the given data in (3.2)-(3.5), it is found that $M_{1} \approx 1.5256638, M_{2} \approx 0.58161945$, $M_{3} \approx 0.258819045, M_{4} \approx 1.26605098$. Clearly $\ell_{1}=1 / 16, \ell_{2}=1 / 16$, and consequently $\left(M_{1}+M_{3}\right) \ell_{1}+\left(M_{2}+M_{4}\right) \ell_{2} \approx 0.22700958<1$.

Thus all the conditions of Theorem 3.2 are satisfied; consequently, its conclusion applies to problem (3.9).

\subsection{Multi-valued system (1.2)-(1.3)}

Definition 3.4 A function $(x, y) \in C^{1}([0, T], \mathbb{R}) \times C^{2}([0, T], \mathbb{R})$ satisfying the coupled boundary conditions $x(0)=\lambda^{c} D^{p} y(\eta), y(0)=0, y(T)=\gamma I^{q} x(\xi)$ and for which there exist functions $f, g \in L^{1}([0, T], \mathbb{R})$ such that $f(t) \in F(t, x(t), y(t)), g(t) \in G(t, x(t), y(t))$ a.e. on $t \in[0, T]$ and

$$
x(t)=I^{\alpha} f(t)+\frac{1}{\Lambda}\left[-\lambda T^{\beta-1} I^{\beta-p} g(\eta)+\lambda \frac{\Gamma(\beta)}{\Gamma(\beta-p)} \eta^{\beta-p-1}\left(\gamma I^{q+\alpha} f(\xi)-I^{\beta} g(T)\right)\right],
$$

and

$$
y(t)=I^{\beta} g(t)+\frac{t^{\beta-1}}{\Lambda}\left[I^{\beta} g(T)-\gamma I^{q+\alpha} f(\xi)-\lambda \gamma \frac{\xi^{q}}{\Gamma(1+q)} I^{\beta-p} g(\eta)\right]
$$

is called a solution of coupled system (1.2)-(1.3).

For each $(x, y) \in X \times X$, the sets of selections of $F, G$ are defined by

$$
S_{F,(x, y)}=\left\{f \in L^{1}([0, T], \mathbb{R}): f(t) \in F(t, x(t), y(t)) \text { for a.e. } t \in[0, T]\right\}
$$

and

$$
S_{G,(x, y)}=\left\{g \in L^{1}([0, T], \mathbb{R}): g(t) \in G(t, x(t), y(t)) \text { for a.e. } t \in[0, T]\right\} .
$$

In view of Lemma 2.5, we define the operators $\mathcal{K}_{1}, \mathcal{K}_{2}: X \times X \rightarrow \mathcal{P}(X \times X)$ as follows:

$$
\begin{aligned}
\mathcal{K}_{1}(x, y)(t)= & \left\{h_{1} \in X \times X: \text { there exist } f \in S_{F,(x, y)}, g \in S_{G,(x, y)}\right. \text { such that } \\
& \left.h_{1}(x, y)(t)=Q_{1}(x, y)(t), \forall t \in[0, T]\right\}
\end{aligned}
$$

and

$$
\begin{aligned}
\mathcal{K}_{2}(x, y)(t)= & \left\{h_{2} \in X \times X: \text { there exists } f \in S_{F,(x, y)}, g \in S_{G,(x, y)}\right. \text { such that } \\
& \left.h_{2}(x, y)(t)=Q_{2}(x, y)(t), \forall t \in[0, T]\right\},
\end{aligned}
$$

where

$$
\begin{aligned}
& Q_{1}(x, y)(t) \\
& \quad=I^{\alpha} f(t)+\frac{1}{\Lambda}\left[-\lambda T^{\beta-1} I^{\beta-p} g(\eta)+\lambda \frac{\Gamma(\beta)}{\Gamma(\beta-p)} \eta^{\beta-p-1}\left(\gamma I^{q+\alpha} f(\xi)-I^{\beta} g(T)\right)\right]
\end{aligned}
$$


and

$$
Q_{2}(x, y)(t)=I^{\beta} g(t)+\frac{t^{\beta-1}}{\Lambda}\left[I^{\beta} g(T)-\gamma I^{q+\alpha} f(\xi)-\lambda \gamma \frac{\xi^{q}}{\Gamma(1+q)} I^{\beta-p} g(\eta)\right] .
$$

Then we define an operator $\mathcal{K}: X \times X \rightarrow \mathcal{P}(X \times X)$ by

$$
\mathcal{K}(x, y)(t)=\left(\begin{array}{l}
\mathcal{K}_{1}(x, y)(t) \\
\mathcal{K}_{2}(x, y)(t)
\end{array}\right)
$$

where $\mathcal{K}_{1}$ and $\mathcal{K}_{2}$ are defined by (3.12) and (3.13).

\subsubsection{The Carathéodory case}

Our first result dealing with convex values $F$ and $G$ is proved via the Leray-Schauder nonlinear alternative for multi-valued maps [37].

Theorem 3.5 Suppose that the following conditions are satisfied:

$\left(B_{1}\right) F, G:[0, T] \times \mathbb{R}^{2} \rightarrow \mathcal{P}(\mathbb{R})$ are $L^{1}$-Carathéodory and have convex values;

$\left(B_{2}\right)$ There exist continuous nondecreasing functions $\psi_{1}, \psi_{2}, \phi_{1}, \phi_{2}:[0, \infty) \rightarrow(0, \infty)$ and functions $p_{1}, p_{2} \in C\left([0, T], \mathbb{R}_{+}\right)$such that

$$
\|F(t, x, y)\|_{\mathcal{P}}:=\sup \{|f|: f \in F(t, x, y)\} \leq p_{1}(t)\left[\psi_{1}(\|x\|)+\phi_{1}(\|y\|)\right]
$$

and

$$
\|G(t, x, y)\|_{\mathcal{P}}:=\sup \{|g|: g \in G(t, x, y)\} \leq p_{2}(t)\left[\psi_{2}(\|x\|)+\phi_{2}(\|y\|)\right]
$$

for each $(t, x, y) \in[0, T] \times \mathbb{R}^{2} ;$

$\left(B_{3}\right)$ There exists a number $N>0$ such that

$$
\frac{N}{\left(M_{1}+M_{3}\right)\left\|p_{1}\right\|\left(\psi_{1}(N)+\phi_{1}(N)\right)+\left(M_{2}+M_{4}\right)\left\|p_{2}\right\|\left(\psi_{2}(N)+\phi_{2}(N)\right)}>1,
$$

where $M_{i}(i=1,2,3,4)$ are given by (3.2)-(3.5).

Then coupled system (1.2)-(1.3) has at least one solution on [0,T].

Proof Consider the operators $\mathcal{K}_{1}, \mathcal{K}_{2}: X \times X \rightarrow \mathcal{P}(X \times X)$ defined by (3.12) and (3.13). From $\left(B_{1}\right)$, it follows that the sets $S_{F,(x, y)}$ and $S_{G,(x, y)}$ are nonempty for each $(x, y) \in X \times X$. Then, for $f \in S_{F,(x, y)}, g \in S_{G,(x, y)}$ for $(x, y) \in X \times X$, we have

$$
h_{1}(x, y)(t)=I^{\alpha} f(t)+\frac{1}{\Lambda}\left[-\lambda T^{\beta-1} I^{\beta-p} g(\eta)+\lambda \frac{\Gamma(\beta)}{\Gamma(\beta-p)} \eta^{\beta-p-1}\left(\gamma I^{q+\alpha} f(\xi)-I^{\beta} g(T)\right)\right]
$$

and

$$
h_{2}(x, y)(t)=I^{\beta} g(t)+\frac{t^{\beta-1}}{\Lambda}\left[I^{\beta} g(T)-\gamma I^{q+\alpha} f(\xi)-\lambda \gamma \frac{\xi^{q}}{\Gamma(1+q)} I^{\beta-p} g(\eta)\right]
$$

where $h_{1} \in \mathcal{K}_{1}(x, y), h_{2} \in \mathcal{K}_{2}(x, y)$, and so $\left(h_{1}, h_{2}\right) \in \mathcal{K}(x, y)$. 
It will be established in several steps that the operator $\mathcal{K}$ satisfies the hypotheses of Leray-Schauder nonlinear alternative. First we show that $\mathcal{K}(x, y)$ is convex valued. Let $\left(h_{i}, \bar{h}_{i}\right) \in\left(\mathcal{K}_{1}, \mathcal{K}_{2}\right), i=1,2$. Then there exist $f_{i} \in S_{F,(x, y)}, g_{i} \in S_{G,(x, y)}, i=1,2$, such that, for each $t \in[0, T]$, we have

$$
h_{i}(t)=I^{\alpha} f(t)+\frac{1}{\Lambda}\left[-\lambda T^{\beta-1} I^{\beta-p} g(\eta)+\lambda \frac{\Gamma(\beta)}{\Gamma(\beta-p)} \eta^{\beta-p-1}\left(\gamma I^{q+\alpha} f(\xi)-I^{\beta} g(T)\right)\right]
$$

and

$$
\bar{h}_{i}(t)=I^{\beta} g(t)+\frac{t^{\beta-1}}{\Lambda}\left[I^{\beta} g(T)-\gamma I^{q+\alpha} f(\xi)-\lambda \gamma \frac{\xi^{q}}{\Gamma(1+q)} I^{\beta-p} g(\eta)\right] .
$$

Let $0 \leq \omega \leq 1$. Then, for each $t \in[0, T]$, we have

$$
\begin{aligned}
& {\left[\omega h_{1}+(1-\omega) h_{2}\right](t)} \\
& =I^{\alpha}\left[\omega f_{1}(s)+(1-\omega) f_{2}(s)\right](t)+\frac{1}{\Lambda}\left[-\lambda T^{\beta-1} I^{\beta-p}\left[\omega g_{1}(s)+(1-\omega) g_{2}(s)\right](\eta)\right. \\
& \quad+\lambda \frac{\Gamma(\beta)}{\Gamma(\beta-p)} \eta^{\beta-p-1}\left(I^{\beta}\left[\omega g_{1}(s)+(1-\omega) g_{2}(s)\right](T)\right. \\
& \left.\left.\quad-\gamma I^{q+\alpha}\left[\omega f_{1}(s)+(1-\omega) f_{2}(s)\right](\xi)\right)\right]
\end{aligned}
$$

and

$$
\begin{aligned}
& {\left[\omega \bar{h}_{1}+(1-\omega) \bar{h}_{2}\right](t)} \\
& =I^{\beta}\left[\omega g_{1}(s)+(1-\omega) g_{2}(s)\right](t)+\frac{t^{\beta-1}}{\Lambda}\left[I^{\beta}\left[\omega g_{1}(s)+(1-\omega) g_{2}(s)\right](T)\right. \\
& \left.\quad-\gamma I^{q+\alpha}\left[\omega f_{1}(s)+(1-\omega) f_{2}(s)\right](\xi)-\lambda \gamma \frac{\xi^{q}}{\Gamma(1+q)} I^{\beta-p}\left[\omega g_{1}(s)+(1-\omega) g_{2}(s)\right](\eta)\right] .
\end{aligned}
$$

We deduce that $S_{F,(x, y)}, S_{G,(x, y)}$ are convex valued, since $F, G$ are convex valued. Obviously, $\omega h_{1}+(1-\omega) h_{2} \in \mathcal{K}_{1}, \omega \bar{h}_{1}+(1-\omega) \bar{h}_{2} \in \mathcal{K}_{2}$, and hence $\omega\left(h_{1}, \bar{h}_{1}\right)+(1-\omega)\left(h_{2}, \bar{h}_{2}\right) \in \mathcal{K}$.

Now we show that $\mathcal{K}$ maps bounded sets into bounded sets in $X \times X$. For a positive number $r$, let $B_{r}=\{(x, y) \in X \times X:\|(x, y)\| \leq r\}$ be a bounded set in $X \times X$. Then there exist $f \in S_{F,(x, y)}, g \in S_{G,(x, y)}$ such that

$$
h_{1}(x, y)(t)=I^{\alpha} f(t)+\frac{1}{\Lambda}\left[-\lambda T^{\beta-1} I^{\beta-p} g(\eta)+\lambda \frac{\Gamma(\beta)}{\Gamma(\beta-p)} \eta^{\beta-p-1}\left(\gamma I^{q+\alpha} f(\xi)-I^{\beta} g(T)\right)\right]
$$

and

$$
h_{2}(x, y)(t)=I^{\beta} g(t)+\frac{t^{\beta-1}}{\Lambda}\left[I^{\beta} g(T)-\gamma I^{q+\alpha} f(\xi)-\lambda \gamma \frac{\xi^{q}}{\Gamma(1+q)} I^{\beta-p} g(\eta)\right] .
$$

Then we have

$$
\begin{aligned}
& \left|h_{1}(x, y)(t)\right| \\
& \quad \leq I^{\alpha}|f(t)|+\frac{1}{|\Lambda|}\left[|\lambda| T^{\beta-1} I^{\beta-p}|g(\eta)|+|\lambda| \frac{\Gamma(\beta)}{\Gamma(\beta-p)} \eta^{\beta-p-1}\left(I^{\beta}|g(T)|+|\gamma| I^{q+\alpha}|f(\xi)|\right)\right]
\end{aligned}
$$




$$
\begin{aligned}
\leq & \frac{T^{\alpha}}{\Gamma(1+\alpha)}\left\|p_{1}\right\|\left(\psi_{1}(r)+\phi_{1}(r)\right)+\frac{1}{|\Lambda|}\left[|\lambda| T^{\beta-1} \frac{\eta^{\beta-p}}{\Gamma(\beta-p+1)}\left\|p_{2}\right\|\left(\psi_{2}(r)+\phi_{2}(r)\right)\right. \\
& +|\lambda| \frac{\Gamma(\beta)}{\Gamma(\beta-p)} \eta^{\beta-p-1}\left(\frac{T^{\beta}}{\Gamma(1+\beta)}\left\|p_{2}\right\|\left(\psi_{2}(r)+\phi_{2}(r)\right)\right. \\
& \left.\left.+|\gamma| \frac{\xi^{q+\alpha}}{\Gamma(q+\alpha+1)}\left\|p_{1}\right\|\left(\psi_{1}(r)+\phi_{1}(r)\right)\right)\right] \\
= & M_{1}\left\|p_{1}\right\|\left(\psi_{1}(r)+\phi_{1}(r)\right)+M_{2}\left\|p_{2}\right\|\left(\psi_{2}(r)+\phi_{2}(r)\right)
\end{aligned}
$$

and

$$
\left|h_{2}(x, y)(t)\right| \leq M_{3}\left\|p_{1}\right\|\left(\psi_{1}(r)+\phi_{1}(r)\right)+M_{4}\left\|p_{2}\right\|\left(\psi_{2}(r)+\phi_{2}(r)\right) .
$$

Thus,

$$
\left\|h_{1}(x, y)\right\| \leq M_{1}\left\|p_{1}\right\|\left(\psi_{1}(r)+\phi_{1}(r)\right)+M_{2}\left\|p_{2}\right\|\left(\psi_{2}(r)+\phi_{2}(r)\right)
$$

and

$$
\left\|h_{2}(x, y)\right\| \leq M_{3}\left\|p_{1}\right\|\left(\psi_{1}(r)+\phi_{1}(r)\right)+M_{4}\left\|p_{2}\right\|\left(\psi_{2}(r)+\phi_{2}(r)\right) .
$$

Hence we obtain

$$
\begin{aligned}
\left\|\left(h_{1}, h_{2}\right)\right\| & =\left\|h_{1}(x, y)\right\|+\left\|h_{2}(x, y)\right\| \\
& \leq\left(M_{1}+M_{3}\right)\left\|p_{1}\right\|\left(\psi_{1}(r)+\phi_{1}(r)\right)+\left(M_{2}+M_{4}\right)\left\|p_{2}\right\|\left(\psi_{2}(r)+\phi_{2}(r)\right) .
\end{aligned}
$$

Next, we show that $\mathcal{K}$ is equicontinuous. Let $t_{1}, t_{2} \in[0, T]$ with $t_{1}<t_{2}$. Then there exist $f \in S_{F,(x, y)}, g \in S_{G,(x, y)}$ such that

$$
h_{1}(x, y)(t)=I^{\alpha} f(t)+\frac{1}{\Lambda}\left[-\lambda T^{\beta-1} I^{\beta-p} g(\eta)+\lambda \frac{\Gamma(\beta)}{\Gamma(\beta-p)} \eta^{\beta-p-1}\left(\gamma I^{q+\alpha} f(\xi)-I^{\beta} g(T)\right)\right]
$$

and

$$
h_{2}(x, y)(t)=I^{\beta} g(t)+\frac{t^{\beta-1}}{\Lambda}\left[I^{\beta} g(T)-\gamma I^{q+\alpha} f(\xi)-\lambda \gamma \frac{\xi^{q}}{\Gamma(1+q)} I^{\beta-p} g(\eta)\right] .
$$

Then we have

$$
\begin{aligned}
& \left|h_{1}(x, y)\left(t_{2}\right)-h_{1}(x, y)\left(t_{1}\right)\right| \\
& \quad \leq\left\|p_{1}\right\|\left(\psi_{1}(r)+\phi_{1}(r)\right)\left|\frac{1}{\Gamma(\alpha)} \int_{0}^{t_{2}}\left(t_{2}-s\right)^{\alpha-1} d s-\frac{1}{\Gamma(\alpha)} \int_{0}^{t_{1}}\left(t_{1}-s\right)^{\alpha-1} d s\right| \\
& \quad \leq \frac{\left\|p_{1}\right\|\left(\psi_{1}(r)+\phi_{1}(r)\right)}{\Gamma(\alpha)}\left\{\int_{0}^{t_{1}}\left[\left(t_{2}-s\right)^{\alpha-1}-\left(t_{1}-s\right)^{\alpha-1}\right] d s+\int_{t_{1}}^{t_{2}}\left(t_{2}-s\right)^{\alpha-1} d s\right\} \\
& \quad \leq \frac{\left\|p_{1}\right\|\left(\psi_{1}(r)+\phi_{1}(r)\right)}{\Gamma(\alpha+1)}\left[2\left(t_{2}-t_{1}\right)^{\alpha}+\left|t_{2}^{\alpha}-t_{1}^{\alpha}\right|\right] .
\end{aligned}
$$


Analogously, we can obtain

$$
\begin{aligned}
& \left|h_{2}(x, y)\left(t_{2}\right)-h_{2}(x, y)\left(t_{1}\right)\right| \\
& \leq\left\|p_{2}\right\|\left(\psi_{2}(r)+\phi_{2}(r)\right)\left[\frac{T^{\beta}}{\Gamma(1+\beta)}+|\lambda||\gamma| \frac{\xi^{q} \eta^{\beta-p}}{\Gamma(1+q) \Gamma(\beta-p+1)}\right] \frac{t_{2}^{\beta-1}-t_{1}^{\beta-1}}{|\Lambda|} \\
& \quad+\left\|p_{1}\right\|\left(\psi_{1}(r)+\phi_{1}(r)\right) \frac{|\gamma| \xi^{q+\alpha}}{\Gamma(q+\alpha+1)} \frac{t_{2}^{\beta-1}-t_{1}^{\beta-1}}{|\Lambda|} \\
& \quad+\frac{\left\|p_{2}\right\|\left(\psi_{2}(r)+\phi_{2}(r)\right)}{\Gamma(\beta+1)}\left[2\left(t_{2}-t_{1}\right)^{\beta}+\left|t_{2}^{\beta}-t_{1}^{\beta}\right|\right] .
\end{aligned}
$$

Therefore, the operator $\mathcal{K}(x, y)$ is equicontinuous, and thus, by the Ascoli-Arzelá theorem, the operator $\mathcal{K}(x, y)$ is completely continuous. We know from [35, Proposition 1.2] that a completely continuous operator is upper semicontinuous if it has a closed graph. Thus we need to prove that $\mathcal{K}$ has a closed graph. Let $\left(x_{n}, y_{n}\right) \rightarrow\left(x_{*}, y_{*}\right),\left(h_{n}, \bar{h}_{n}\right) \in \mathcal{K}\left(x_{n}, y_{n}\right)$ and $\left(h_{n}, \bar{h}_{n}\right) \rightarrow\left(h_{*}, \bar{h}_{*}\right)$, then we need to show $\left(h_{*}, \bar{h}_{*}\right) \in \mathcal{K}\left(x_{*}, y_{*}\right)$. Observe that $\left(h_{n}, \bar{h}_{n}\right) \in$ $\mathcal{K}\left(x_{n}, y_{n}\right)$ implies that there exist $f_{n} \in S_{F,\left(x_{n}, y_{n}\right)}$ and $g_{n} \in S_{G,\left(x_{n}, y_{n}\right)}$ such that

$$
\begin{aligned}
& h_{n}\left(x_{n}, y_{n}\right)(t) \\
& \quad=I^{\alpha} f_{n}(t)+\frac{1}{\Lambda}\left[-\lambda T^{\beta-1} I^{\beta-p} g_{n}(\eta)+\lambda \frac{\Gamma(\beta)}{\Gamma(\beta-p)} \eta^{\beta-p-1}\left(\gamma I^{q+\alpha} f_{n}(\xi)-I^{\beta} g_{n}(T)\right)\right]
\end{aligned}
$$

and

$$
\bar{h}_{n}\left(x_{n}, y_{n}\right)(t)=I^{\beta} g_{n}(t)+\frac{t^{\beta-1}}{\Lambda}\left[I^{\beta} g_{n}(T)-\gamma I^{q+\alpha} f_{n}(\xi)-\lambda \gamma \frac{\xi^{q}}{\Gamma(1+q)} I^{\beta-p} g_{n}(\eta)\right] .
$$

Let us consider the continuous linear operators $\Phi_{1}, \Phi_{2}: L^{1}([0, T], X \times X) \rightarrow C([0, T], X \times$ $X)$ given by

$$
\Phi_{1}(x, y)(t)=I^{\alpha} f(t)+\frac{1}{\Lambda}\left[-\lambda T^{\beta-1} I^{\beta-p} g(\eta)+\lambda \frac{\Gamma(\beta)}{\Gamma(\beta-p)} \eta^{\beta-p-1}\left(\gamma I^{q+\alpha} f(\xi)-I^{\beta} g(T)\right)\right]
$$

and

$$
\Phi_{2}(x, y)(t)=I^{\beta} g(t)+\frac{t^{\beta-1}}{\Lambda}\left[I^{\beta} g(T)-\gamma I^{q+\alpha} f(\xi)-\lambda \gamma \frac{\xi^{q}}{\Gamma(1+q)} I^{\beta-p} g(\eta)\right] .
$$

From [38] we know that $\left(\Phi_{1}, \Phi_{2}\right) \circ\left(S_{F}, S_{G}\right)$ is a closed graph operator. Further, we have $\left(h_{n}, \bar{h}_{n}\right) \in\left(\Phi_{1}, \Phi_{2}\right) \circ\left(S_{F,\left(x_{n}, y_{n}\right)}, S_{G,\left(x_{n}, y_{n}\right)}\right)$ for all $n$. Since $\left(x_{n}, y_{n}\right) \rightarrow\left(x_{*}, y_{*}\right),\left(h_{n}, \bar{h}_{n}\right) \rightarrow\left(h_{*}, \bar{h}_{*}\right)$ it follows that $f_{*} \in S_{F,(x, y)}$ and $g_{*} \in S_{G,(x, y)}$ such that

$$
\begin{aligned}
& h_{*}\left(x_{*}, y_{*}\right)(t) \\
& \quad=I^{\alpha} f_{*}(t)+\frac{1}{\Lambda}\left[-\lambda T^{\beta-1} I^{\beta-p} g_{*}(\eta)+\lambda \frac{\Gamma(\beta)}{\Gamma(\beta-p)} \eta^{\beta-p-1}\left(\gamma I^{q+\alpha} f_{*}(\xi)-I^{\beta} g_{*}(T)\right)\right]
\end{aligned}
$$

and

$$
\bar{h}_{*}\left(x_{*}, y_{*}\right)(t)+I^{\beta} g_{*}(t)+\frac{t^{\beta-1}}{\Lambda}\left[I^{\beta} g_{*}(T)-\gamma I^{q+\alpha} f_{*}(\xi)-\lambda \gamma \frac{\xi^{q}}{\Gamma(1+q)} I^{\beta-p} g_{*}(\eta)\right],
$$

that is, $\left(h_{n}, \bar{h}_{n}\right) \in \mathcal{K}\left(x_{*}, y_{*}\right)$. 
Finally, we establish the a priori bounds on solutions. Let $(x, y) \in \nu \mathcal{K}(x, y)$. Then there exist $f \in S_{F,(x, y)}$ and $g \in S_{G,(x, y)}$ such that

$$
x(t)=v I^{\alpha} f(t)+\nu \frac{1}{\Lambda}\left[-\lambda T^{\beta-1} I^{\beta-p} g(\eta)+\lambda \frac{\Gamma(\beta)}{\Gamma(\beta-p)} \eta^{\beta-p-1}\left(\gamma I^{q+\alpha} f(\xi)-I^{\beta} g(T)\right)\right]
$$

and

$$
y(t)=v I^{\beta} g(t)+\nu \frac{t^{\beta-1}}{\Lambda}\left[I^{\beta} g(T)-\gamma I^{q+\alpha} f(\xi)-\lambda \gamma \frac{\xi^{q}}{\Gamma(1+q)} I^{\beta-p} g(\eta)\right] .
$$

For each $t \in[0, T]$, we obtain

$$
\|x\| \leq M_{1}\left\|p_{1}\right\|\left(\psi_{1}(\|x\|)+\phi_{1}(\|y\|)\right)+M_{2}\left\|p_{2}\right\|\left(\psi_{2}(\|x\|)+\phi_{2}(\|y\|)\right)
$$

and

$$
\|y\| \leq M_{3}\left\|p_{1}\right\|\left(\psi_{1}(\|x\|)+\phi_{1}(\|y\|)\right)+M_{4}\left\|p_{2}\right\|\left(\psi_{2}(\|x\|)+\phi_{2}(\|y\|)\right),
$$

following the same arguments as in the second step.

Thus

$$
\begin{aligned}
\|(x, y)\|= & \|x\|+\|y\| \\
\leq & \left(M_{1}+M_{3}\right)\left\|p_{1}\right\|\left(\psi_{1}(\|x\|)+\phi_{1}(\|y\|)\right) \\
& +\left(M_{2}+M_{4}\right)\left\|p_{2}\right\|\left(\psi_{2}(\|x\|)+\phi_{2}(\|y\|)\right),
\end{aligned}
$$

which implies that

$$
\frac{\|(x, y)\|}{\left(M_{1}+M_{3}\right)\left\|p_{1}\right\|\left(\psi_{1}(\|x\|)+\phi_{1}(\|y\|)\right)+\left(M_{2}+M_{4}\right)\left\|p_{2}\right\|\left(\psi_{2}(\|x\|)+\phi_{2}(\|y\|)\right)} \leq 1 .
$$

In view of $\left(B_{3}\right)$, there exists $N$ such that $\|(x, y)\| \neq N$. Let us set

$$
U=\{(x, y) \in X \times X:\|(x, y)\|<N\} .
$$

Note that the operator $\mathcal{K}: \bar{U} \rightarrow \mathcal{P}_{c p, c v}(X) \times \mathcal{P}_{c p, c v}(X)$ is completely continuous and upper semicontinuous. There is no $(x, y) \in \partial U$ such that $(x, y) \in v \mathcal{K}(x, y)$ for some $v \in(0,1)$ by the choice of $U$. Hence, by the nonlinear alternative of Leray-Schauder type [37], we deduce that $\mathcal{K}$ has a fixed point $(x, y) \in \bar{U}$, which is a solution of coupled system (1.2)-(1.3). This completes the proof.

\subsubsection{The Lipschitz case}

This subsection is concerned with the case when the multi-valued maps in system (1.2) have non-convex values.

Let $(X, d)$ be a metric space induced from the normed space $(X ;\|\cdot\|)$, and let $H_{d}$ : $\mathcal{P}(X) \times \mathcal{P}(X) \rightarrow \mathbb{R} \cup\{\infty\}$ be defined by $H_{d}(U, V)=\max \left\{\sup _{u \in U} d(u, V), \sup _{v \in V} d(U, v)\right\}$, where $d(U, v)=\inf _{u \in U} d(u, v)$ and $d(u, V)=\inf _{v \in V} d(u, v)$. Then $\left(\mathcal{P}_{b, c l}(X), H_{d}\right)$ is a metric space and $\left(\mathcal{P}_{c l}(X), H_{d}\right)$ is a generalized metric space (see [39]). 
Definition 3.6 A multi-valued operator $\mathcal{G}: X \rightarrow \mathcal{P}_{c l}(X)$ is called (i) $\gamma$-Lipschitz if and only if there exists $\gamma>0$ such that $H_{d}(\mathcal{G}(a), \mathcal{G}(b)) \leq \gamma d(a, b)$ for each $a, b \in X$; and (ii) a contraction if and only if it is $\gamma$-Lipschitz with $\gamma<1$.

In the forthcoming result, we make use of the fixed point theorem for multi-valued maps due to Covitz and Nadler [40].

\section{Theorem 3.7 If}

$\left(B_{3}\right) F, G:[0, T] \times \mathbb{R}^{2} \rightarrow \mathcal{P}_{c p}(\mathbb{R})$ are such that $F(\cdot, x, y):[0, T] \rightarrow \mathcal{P}_{c p}(\mathbb{R})$ and $G(\cdot, x, y):$ $[0, T] \rightarrow \mathcal{P}_{c p}(\mathbb{R})$ are measurable for each $x, y \in \mathbb{R} ;$

$\left(B_{4}\right)$

$$
H_{d}\left(F(t, x, y), F(t, \bar{x}, \bar{y}) \leq m_{1}(t)(|x-\bar{x}|+|y-\bar{y}|)\right.
$$

and

$$
H_{d}\left(G(t, x, y), G(t, \bar{x}, \bar{y}) \leq m_{2}(t)(|x-\bar{x}|+|y-\bar{y}|)\right.
$$

for almost all $t \in[0, T]$ and $x, y, \bar{x}, \bar{y} \in \mathbb{R}$ with $m_{1}, m_{2} \in C\left([0, T], \mathbb{R}^{+}\right)$and $d(0, F(t$, $0,0)) \leq m_{1}(t), d(0, G(t, 0,0)) \leq m_{2}(t)$ for almost all $t \in[0, T]$

hold, then coupled system (1.2)-(1.3) has at least one solution on $[0, T]$ provided that

$$
\left(M_{1}+M_{3}\right)\left\|m_{1}\right\|+\left(M_{2}+M_{4}\right)\left\|m_{2}\right\|<1 .
$$

Proof The sets $S_{F,(x, y)}$ and $S_{G,(x, y)}$ are nonempty for each $(x, y) \in X \times Y$ by assumption $\left(B_{3}\right)$, so $F$ and $G$ have measurable selections (see Theorem III.6 in [41]). Now we show that the operator $\mathcal{K}$ satisfies the assumptions of Covitz and Nadler's fixed point theorem [40].

First we show that $\mathcal{K}(x, y) \in \mathcal{P}_{c l}(X) \times \mathcal{P}_{c l}(X)$ for each $(x, y) \in X \times X$. Let $\left(h_{n}, \bar{h}_{n}\right) \in \mathcal{K}\left(x_{n}, y_{n}\right)$ such that $\left(h_{n}, \bar{h}_{n}\right) \rightarrow(h, \bar{h})$ in $X \times X$. Then $(h, \bar{h}) \in X \times X$ and there exist $f_{n} \in S_{F,\left(x_{n}, y_{n}\right)}$ and $g_{n} \in S_{G,\left(x_{n}, y_{n}\right)}$ such that

$$
\begin{aligned}
& h_{n}\left(x_{n}, y_{n}\right)(t) \\
& \quad=I^{\alpha} f_{n}(t)+\frac{1}{\Lambda}\left[-\lambda T^{\beta-1} I^{\beta-p} g_{n}(\eta)+\lambda \frac{\Gamma(\beta)}{\Gamma(\beta-p)} \eta^{\beta-p-1}\left(I^{\beta} g_{n}(T)-\gamma I^{q+\alpha} f_{n}(\xi)\right)\right]
\end{aligned}
$$

and

$$
\bar{h}_{n}\left(x_{n}, y_{n}\right)(t)=I^{\beta} g_{n}(t)+\frac{t^{\beta-1}}{\Lambda}\left[I^{\beta} g_{n}(T)-\gamma I^{q+\alpha} f_{n}(\xi)-\lambda \gamma \frac{\xi^{q}}{\Gamma(1+q)} I^{\beta-p} g_{n}(\eta)\right] .
$$

Since $F$ and $G$ have compact values, we pass onto subsequences (denoted as sequences) to get that $f_{n}$ and $g_{n}$ converge to $f$ and $g$ in $L^{1}([0, T], \mathbb{R})$ respectively. Thus $f \in S_{F,(x, y)}$ and $g \in S_{G,(x, y)}$ for each $t \in[0, T]$ and that

$$
\begin{aligned}
h_{n}\left(x_{n}, y_{n}\right)(t) & \rightarrow h(x, y)(t) \\
& =I^{\alpha} f(t)+\frac{1}{\Lambda}\left[-\lambda T^{\beta-1} I^{\beta-p} g(\eta)+\lambda \frac{\Gamma(\beta)}{\Gamma(\beta-p)} \eta^{\beta-p-1}\left(\gamma I^{q+\alpha} f(\xi)-I^{\beta} g(T)\right)\right],
\end{aligned}
$$


and

$$
\begin{aligned}
\bar{h}_{n}\left(x_{n}, y_{n}\right)(t) & \rightarrow \bar{h}(x, y)(t) \\
& =I^{\beta} g(t)+\frac{t^{\beta-1}}{\Lambda}\left[I^{\beta} g(T)-\gamma I^{q+\alpha} f(\xi)-\lambda \gamma \frac{\xi^{q}}{\Gamma(1+q)} I^{\beta-p} g(\eta)\right] .
\end{aligned}
$$

Hence $(h, \bar{h}) \in \mathcal{K}$, which implies that $\mathcal{K}$ is closed.

Next we show that there exists $\widehat{\theta}<1$ (defined by (3.14)) such that

$$
H_{d}(\mathcal{K}(x, y), \mathcal{K}(\bar{x}, \bar{y})) \leq \widehat{\theta}(\|x-\bar{x}\|+\|y-\bar{y}\|) \quad \text { for each } x, \bar{x}, y, \bar{y} \in X
$$

Let $(x, \bar{x}),(y, \bar{y}) \in X \times X$ and $\left(h_{1}, \overline{h_{1}}\right) \in \mathcal{K}(x, y)$. Then there exist $f_{1} \in S_{F,(x, y)}$ and $g_{1} \in S_{G,(x, y)}$ such that, for each $t \in[0, T]$, we have

$$
\begin{aligned}
& h_{1}\left(x_{n}, y_{n}\right)(t) \\
& \quad=I^{\alpha} f_{1}(t)+\frac{1}{\Lambda}\left[-\lambda T^{\beta-1} I^{\beta-p} g_{1}(\eta)+\lambda \frac{\Gamma(\beta)}{\Gamma(\beta-p)} \eta^{\beta-p-1}\left(I^{\beta} g_{1}(T)-\gamma I^{q+\alpha} f_{1}(\xi)\right)\right]
\end{aligned}
$$

and

$$
\bar{h}_{1}\left(x_{n}, y_{n}\right)(t)=I^{\beta} g_{1}(t)+\frac{t^{\beta-1}}{\Lambda}\left[I^{\beta} g_{1}(T)-\gamma I^{q+\alpha} f_{1}(\xi)-\lambda \gamma \frac{\xi^{q}}{\Gamma(1+q)} I^{\beta-p} g_{1}(\eta)\right] .
$$

By $\left(B_{4}\right)$, we have

$$
H_{d}(F(t, x, y), F(t, \bar{x}, \bar{y})) \leq m_{1}(t)(|x(t)-\bar{x}(t)|+|y(t)-\bar{y}(t)|)
$$

and

$$
H_{d}(G(t, x, y), G(t, \bar{x}, \bar{y})) \leq m_{2}(t)(|x(t)-\bar{x}(t)|+|y(t)-\bar{y}(t)|) .
$$

So, there exist $f \in F(t, x(t), y(t))$ and $g \in G(t, x(t), y(t))$ such that

$$
\left|f_{1}(t)-w\right| \leq m_{1}(t)(|x(t)-\bar{x}(t)|+|y(t)-\bar{y}(t)|)
$$

and

$$
\left|g_{1}(t)-z\right| \leq m_{2}(t)(|x(t)-\bar{x}(t)|+|y(t)-\bar{y}(t)|) .
$$

Define $V_{1}, V_{2}:[0, T] \rightarrow \mathcal{P}(\mathbb{R})$ by

$$
V_{1}(t)=\left\{f \in L^{1}([0, T], \mathbb{R}):\left|f_{1}(t)-w\right| \leq m_{1}(t)(|x(t)-\bar{x}(t)|+|y(t)-\bar{y}(t)|)\right\}
$$

and

$$
V_{2}(t)=\left\{g \in L^{1}([0, T], \mathbb{R}):\left|g_{1}(t)-z\right| \leq m_{2}(t)(|x(t)-\bar{x}(t)|+|y(t)-\bar{y}(t)|)\right\} .
$$


Since the multi-valued operators $V_{1}(t) \cap F(t, x(t), y(t))$ and $V_{2}(t) \cap G(t, x(t), y(t))$ are measurable (Proposition III.4 in [41]), there exist functions $f_{2}(t), g_{2}(t)$ which are a measurable selection for $V_{1}, V_{2}$ and $f_{2}(t) \in F(t, x(t), y(t)), g_{2}(t) \in G(t, x(t), y(t))$ such that, for a.e. $t \in[0, T]$, we have

$$
\left|f_{1}(t)-f_{2}(t)\right| \leq m_{1}(t)(|x(t)-\bar{x}(t)|+|y(t)-\bar{y}(t)|)
$$

and

$$
\left|g_{1}(t)-g_{2}(t)\right| \leq m_{g}(t)(|x(t)-\bar{x}(t)|+|y(t)-\bar{y}(t)|) .
$$

Let

$$
\begin{aligned}
& h_{2}\left(x_{n}, y_{n}\right)(t) \\
& \quad=I^{\alpha} f_{2}(t)+\frac{1}{\Lambda}\left[-\lambda T^{\beta-1} I^{\beta-p} g_{2}(\eta)+\lambda \frac{\Gamma(\beta)}{\Gamma(\beta-p)} \eta^{\beta-p-1}\left(I^{\beta} g_{2}(T)-\gamma I^{q+\alpha} f_{2}(\xi)\right)\right]
\end{aligned}
$$

and

$$
\bar{h}_{2}\left(x_{n}, y_{n}\right)(t)=I^{\beta} g_{2}(t)+\frac{t^{\beta-1}}{\Lambda}\left[I^{\beta} g_{2}(T)-\gamma I^{q+\alpha} f_{2}(\xi)-\lambda \gamma \frac{\xi^{q}}{\Gamma(1+q)} I^{\beta-p} g_{2}(\eta)\right] .
$$

Thus,

$$
\begin{aligned}
& \left|h_{1}(x, y)(t)-h_{2}(x, y)(t)\right| \\
& \leq I^{\alpha}\left|f_{1}(s)-f_{2}(s)\right|(t)+\frac{1}{|\Lambda|}\left[|\lambda| T^{\beta-1} I^{\beta-p}\left|g_{1}(s)-g_{2}(s)\right|(\eta)\right. \\
& \left.\quad+|\lambda| \frac{\Gamma(\beta)}{\Gamma(\beta-p)} \eta^{\beta-p-1}\left(I^{\beta}\left|g_{1}(s)-g_{2}(s)\right|(T)+|\gamma| I^{q+\alpha}\left|f_{1}(s)-f_{2}(s)\right|(\xi)\right)\right] \\
& \leq I^{\alpha} m_{1}(s)(|x(s)-\bar{x}(s)|+|y(s)-\bar{y}(s)|)(t) \\
& \quad+\frac{1}{|\Lambda|}\left[|\lambda| T^{\beta-1} I^{\beta-p} m_{2}(s)(|x(s)-\bar{x}(s)|+|y(s)-\bar{y}(s)|)(\eta)\right. \\
& \quad+|\lambda| \frac{\Gamma(\beta)}{\Gamma(\beta-p)} \eta^{\beta-p-1}\left(I^{\beta} m_{2}(s)(|x(s)-\bar{x}(s)|+|y(s)-\bar{y}(s)|)(T)\right. \\
& \left.\left.\quad+|\gamma| I^{q+\alpha} m_{1}(s)(|x(s)-\bar{x}(s)|+|y(s)-\bar{y}(s)|)(\xi)\right)\right] \\
& \leq M_{1}\left\|m_{1}\right\|(\|x-\bar{x}\|+\|y-\bar{y}\|)+M_{2}\left\|m_{2}\right\|(\|x-\bar{x}\|+\|y-\bar{y}\|) .
\end{aligned}
$$

Hence

$$
\left\|h_{1}(x, y)-h_{2}(x, y)\right\| \leq\left(M_{1}\left\|m_{1}\right\|+M_{2}\left\|m_{2}\right\|\right)(\|x-\bar{x}\|+\|y-\bar{y}\|) .
$$

In a similar manner, we can establish that

$$
\left\|\bar{h}_{1}(x, y)-\bar{h}_{2}(x, y)\right\| \leq\left(M_{3}\left\|m_{1}\right\|+M_{4}\left\|m_{2}\right\|\right)(\|x-\bar{x}\|+\|y-\bar{y}\|) .
$$


Thus

$$
\left\|\left(h_{1}, \bar{h}_{1}\right),\left(h_{2}, \bar{h}_{2}\right)\right\| \leq\left[\left(M_{1}+M_{3}\right)\left\|m_{1}\right\|+\left(M_{2}+M_{4}\right)\left\|m_{2}\right\|\right](\|x-\bar{x}\|+\|y-\bar{y}\|) .
$$

Analogously, interchanging the roles of $(x, y)$ and $(\bar{x}, \bar{y})$, we can obtain

$$
H_{d}(T(x, y), T(\bar{x}, \bar{y})) \leq\left[\left(M_{1}+M_{3}\right)\left\|m_{1}\right\|+\left(M_{2}+M_{4}\right)\left\|m_{2}\right\|\right](\|x-\bar{x}\|+\|y-\bar{y}\|) .
$$

Therefore $\mathcal{K}$ is a contraction in view of assumption (3.14). Hence it follows by Covitz and Nadler's fixed point theorem [40] that $\mathcal{K}$ has a fixed point $(x, y)$, which is a solution of problem (1.2)-(1.3). This completes the proof.

\section{Conclusion}

In the present research we studied the existence of solutions for coupled fractional differential equations and inclusions involving fractional derivatives of different orders and supplemented with nonlocal boundary conditions containing fractional derivative and integral. In the single-valued case we establish existence and uniqueness of solutions by applying the Leray-Schauder alternative and the Banach contraction mapping principle respectively. In the multi-valued case we proved existence results for both convex and non-convex multi-valued maps via the nonlinear alternative for Kakutani maps and Covitz and Nadler's fixed point theorem. Examples illustrating the obtained results are also constructed.

\section{Acknowledgements}

This project was funded by the Deanship of Scientific Research (DSR), King Abdulaziz University, Jeddah, under grant No. (D-310-130-1441). The authors, therefore, gratefully acknowledge DSR technical and financial support. We also thank the reviewers for their useful comments on our work.

\section{Funding}

This project was funded by the Deanship of Scientific Research (DSR), King Abdulaziz University, Jeddah, Saudi Arabia under grant no. (D-310-130-1441).

\section{Abbreviations}

Not applicable.

Availability of data and materials

Not applicable.

Competing interests

The authors declare that they have no competing interests.

Authors' contributions

Each of the authors, SKN and HHA, contributed equally to each part of this work. All authors read and approved the final manuscript.

\section{Author details}

${ }^{1}$ Department of Mathematics, University of loannina, loannina, Greece. ${ }^{2}$ Nonlinear Analysis and Applied Mathematics (NAAM) - Research Group, Department of Mathematics, Faculty of Science, King Abdulaziz University, Jeddah, Saudi Arabia.

\section{Publisher's Note}

Springer Nature remains neutral with regard to jurisdictional claims in published maps and institutional affiliations. 


\section{References}

1. Podlubny, I.: Fractional Differential Equations. Academic Press, San Diego (1999)

2. Kilbas, A.A., Srivastava, H.M., Trujillo, J.J.: Theory and Applications of Fractional Differential Equations. North-Holland Mathematics Studies, vol. 204. Elsevier, Amsterdam (2006)

3. Ahmad, B., Alsaedi, A., Ntouyas, S.K., Tariboon, J.: Hadamard-Type Fractional Differential Equations, Inclusions and Inequalities. Springer, Cham (2017)

4. Liang, S., Zhang, J.: Existence of multiple positive solutions for $m$-point fractional boundary value problems on an infinite interval. Math. Comput. Model. 54, 1334-1346 (2011)

5. Wang, J.R., Zhou, Y., Feckan, M.: On recent developments in the theory of boundary value problems for impulsive fractional differential equations. Comput. Math. Appl. 64, 3008-3020 (2012)

6. Zhai, C., Xu, L.: Properties of positive solutions to a class of four-point boundary value problem of Caputo fractional differential equations with a parameter. Commun. Nonlinear Sci. Numer. Simul. 19, 2820-2827 (2014)

7. Henderson, J., Kosmatov, N.: Eigenvalue comparison for fractional boundary value problems with the Caputo derivative. Fract. Calc. Appl. Anal. 17, 872-880 (2014)

8. Ding, Y., Wei, Z., Xu, J., O'Regan, D.: Extremal solutions for nonlinear fractional boundary value problems with p-Laplacian. J. Comput. Appl. Math. 288, 151-158 (2015)

9. Wang, H.: Existence of solutions for fractional anti-periodic BVP. Results Math. 68, 227-245 (2015)

10. Ahmad, B., Ntouyas, S.K.: Some fractional-order one-dimensional semi-linear problems under nonlocal integral boundary conditions. Rev. R. Acad. Cienc. Exactas Fís. Nat., Ser. A Mat. 110, 159-172 (2016)

11. Zhou, Y., Ahmad, B., Alsaedi, A.: Existence of nonoscillatory solutions for fractional neutral differential equations. Appl. Math. Lett. 72, 70-74 (2017)

12. Ge, Z.M., Ou, C.Y.: Chaos synchronization of fractional order modified Duffing systems with parameters excited by a chaotic signal. Chaos Solitons Fractals 35, 705-717 (2008)

13. Faieghi, M., Kuntanapreeda, S., Delavari, H., Baleanu, D.: LMI-based stabilization of a class of fractional-order chaotic systems. Nonlinear Dyn. 72, 301-309 (2013)

14. Zhang, F., Chen, G., Li, C., Kurths, J.: Chaos synchronization in fractional differential systems. Philos. Trans. R. Soc. A, Math. Phys. Eng. Sci. 371, 20120155 (2013)

15. Sokolov, I.M., Klafter, J., Blumen, A.: Fractional kinetics. Phys. Today 55, 48-54 (2002)

16. Javidi, M., Ahmad, B.: Dynamic analysis of time fractional order phytoplankton-toxic phytoplankton-zooplankton system. Ecol. Model. 318, 8-18 (2015)

17. Petras, I., Magin, R.L.: Simulation of drug uptake in a two compartmental fractional model for a biological system. Commun. Nonlinear Sci. Numer. Simul. 16, 4588-4595 (2011)

18. Ding, Y., Wang, Z., Ye, H.: Optimal control of a fractional-order HIV-immune system with memory. IEEE Trans. Control Syst. Technol. 20, 763-769 (2012)

19. Carvalho, A., Pinto, C.M.A.: A delay fractional order model for the co-infection of malaria and HIV/AIDS. Int. J. Dyn. Control 5, 168-186 (2017)

20. Ntouyas, S.K., Obaid, M.: A coupled system of fractional differential equations with nonlocal integral boundary conditions. Adv. Differ. Equ. 2012, 130 (2012)

21. Ahmad, B., Ntouyas, S.K.: Existence results for a coupled system of Caputo type sequential fractional differential equations with nonlocal integral boundary conditions. Appl. Math. Comput. 266, 615-622 (2015)

22. Wang, J.R., Zhang, Y.: Analysis of fractional order differential coupled systems. Math. Methods Appl. Sci. 38, 3322-3338 (2015)

23. Tariboon, J., Ntouyas, S.K., Sudsutad, W.: Coupled systems of Riemann-Liouville fractional differential equations with Hadamard fractional integral boundary conditions. J. Nonlinear Sci. Appl. 9, 295-308 (2016)

24. Ahmad, B., Ntouyas, S.K., Alsaedi, A.: On a coupled system of fractional differential equations with coupled nonloca and integral boundary conditions. Chaos Solitons Fractals 83, 234-241 (2016)

25. Agarwal, R.P., Ahmad, B., Garout, D., Alsaedi, A.: Existence results for coupled nonlinear fractional differential equations equipped with nonlocal coupled flux and multi-point boundary conditions. Chaos Solitons Fractals 102, 149-161 (2017)

26. Ahmad, B., Luca, R.: Existence of solutions for a sequential fractional integro-differential system with coupled integral boundary conditions. Chaos Solitons Fractals 104, 378-388 (2017)

27. Alsulami, H.H., Ntouyas, S.K., Agarwal, R.P., Ahmad, B., Alsaedi, A.: A study of fractional-order coupled systems with a new concept of coupled non-separated boundary conditions. Bound. Value Probl. 2017, 68 (2017)

28. Mahmudov, N.I., Bawaneh, S., Al-Khateeb, A.: On a coupled system of fractional differential equations with four point integral boundary conditions. Mathematics 7, 279 (2019). https://doi.org/10.3390/math7030279

29. Mahmudov, N.I., Al-Khateeb, A.: Stability, existence and uniqueness of boundary value problems for a coupled system of fractional differential equations. Mathematics 7, 354 (2019). https://doi.org/10.3390/math7040354

30. Ntouyas, S.K.: Boundary value problems for nonlinear fractional differential equations and inclusions with nonlocal and fractional integral boundary conditions. Opusc. Math. 33, 117-138 (2013)

31. Ahmad, B., Ntouyas, S.K.: Existence of solutions for fractional differential inclusions with four-point nonlocal Riemann-Liouville type integral boundary conditions. Filomat 27(6), 1027-1036 (2013)

32. Ahmad, B., Ntouyas, S.K., Zhou, Y., Alsaedi, A.: A study of fractional differential equations and inclusions with nonlocal Erdélyi-Kober type integral boundary conditions. Bull. Iran. Math. Soc. 44, 1315-1328 (2018)

33. Ahmad, B., Alghanmi, M., Ntouyas, S.K., Alsaedi, A.: A study of fractional differential equations and inclusions involving generalized Caputo-type derivative equipped with generalized fractional integral boundary conditions. AIMS Math. 4, 12-28 (2019)

34. Ahmad, B., Ntouyas, S.K., Alsaedi, A.: A study of coupled systems of fractional differential inclusions with non-separated coupled boundary conditions. Electron. J. Differ. Equ. 2019, 69 (2019)

35. Deimling, K.: Multivalued Differential Equations. de Gruyter, Berlin (1992)

36. Hu, S., Papageorgiou, N.: Handbook of Multivalued Analysis, Theory I. Kluwer Academic, Dordrecht (1997)

37. Granas, A., Dugundji, J.: Fixed Point Theory. Springer, New York (2005)

38. Lasota, A., Opial, Z:: An application of the Kakutani-Ky Fan theorem in the theory of ordinary differential equations. Bull. Acad. Pol. Sci., Sér. Sci. Math. Astron. Phys. 13, 781-786 (1965) 
39. Kisielewicz, M.: Stochastic Differential Inclusions and Applications. Springer Optimization and Its Applications, vol. 80. Springer, New York (2013)

40. Covitz, H., Nadler, S.B. Jr.: Multivalued contraction mappings in generalized metric spaces. Isr. J. Math. 8, 5-11 (1970)

41. Castaing, C., Valadier, M.: Convex Analysis and Measurable Multifunctions. Lecture Notes in Mathematics, vol. 580. Springer, Berlin (1977)

Submit your manuscript to a SpringerOpen ${ }^{\odot}$ journal and benefit from:

- Convenient online submission

$\checkmark$ Rigorous peer review

- Open access: articles freely available online

- High visibility within the field

- Retaining the copyright to your article

Submit your next manuscript at $\gg$ springeropen.com 